\title{
¿La detección de toxinas de Clostridioides difficile es necesaria cuando se detecta la enzima glutamato deshidrogenasa?
}

\author{
José F. García-Fuentes, ${ }^{1}$ Brenda J. Torres-Murillo, ${ }^{1}$ Gilberto Aguilar-Orozco, ${ }^{2}$ Élida González, ${ }^{2}$ \\ Juan L. Mosqueda, ${ }^{1}$ Alejandro E. Macías ${ }^{1}$ y José A. Álvarez ${ }^{1 *}$ \\ ${ }^{1}$ Universidad de Guanajuato, Departamento de Medicina y Nutrición, Laboratorio de Microbiología; ${ }^{2}$ Hospital Aranda de la Parra. Guanajuato, \\ México
}

\section{Resumen}

Introducción: Clostridioides difficile causa diarrea y colitis pseudomembranosa. Su diagnóstico se realiza con la detección de glutamato-deshidrogenasa (GDH) o las toxinas $A$ y $B$ y se confirma con pruebas de amplificación de ácidos nucleicos. Objetivo: Definir si la determinación de GDH es redundante a la de las toxinas. Métodos: Estudio observacional retrospectivo de muestras fecales de pacientes con sospecha de infección por Clostridioides difficile. Las toxinas y GDH se determinaron mediante inmunocromatografía. Se realizó una simulación bayesiana con los cocientes de probabilidad; se consideró significativo un valor de $p<0.05$. Resultados: Se analizaron 329 resultados de GDH y toxinas $A$ y B. Se encontró una prevalencia de infección de Clostridioides difficile de $18.2 \%$. La sensibilidad y especificidad de la prueba de GDH fue de 0.90 y 0.89 , respectivamente. El cociente de probabilidad positivo fue de 8.9 y el negativo, de 0.11 . Conclusiones: Un resultado negativo de GDH disminuye considerablemente la probabilidad de infección, pero no la descarta. La detección de toxinas de Clostridioides difficile puede ser necesaria en instituciones donde la amplificación de ácidos nucleicos no es económica o accesible.

PALABRAS CLAVE: Clostridioides difficile. Glutamato deshidrogenasa. Toxina A. Toxina B.

\section{Is Clostridioides difficile toxins detection necessary when the glutamate dehydrogenase enzyme is detected?}

\begin{abstract}
Introduction: Clostridioides difficile causes diarrhea and pseudomembranous colitis. Its diagnosis is made with glutamate dehydrogenase $(G D H)$ or toxins $A$ and $B$ detection and is confirmed with nucleic acid amplification tests. Objective: To define if GDH determination is redundant to that of toxins. Methods: Retrospective, observational study in diarrheal stools of patients with suspected Clostridioides difficile infection. Toxins and GDH were determined by immunochromatography. Bayesian simulation was performed with likelihood ratios; a p-value $<0.05$ was regarded as significant. Results: 329 GDH and toxin $A$ and $B$ results were analyzed. Clostridioides difficile infection prevalence was $18.2 \%$. Sensitivity and specificity of the GDH test were 0.90 and 0.89 , respectively. Positive likelihood ratio was 8.9, and negative was 0.11 . Conclusions: $A$ negative GDH result considerably reduces the probability of infection but does not rule it out. Clostridioides difficile toxins detection may be necessary in institutions where nucleic acid amplification is not affordable or accessible.
\end{abstract}

KEY WORDS: Clostridioides difficile. Glutamate dehydrogenase. Toxin A. Toxin B.

Correspondencia:

*José A. Álvarez

E-mail: alvarez_ja@me.com
Gac Med Mex. 2021;157:113-115

Disponible en PubMed

www.gacetamedicademexico.com

0016-3813/@ 2020 Academia Nacional de Medicina de México, A.C. Publicado por Permanyer. Este es un artículo open access bajo la licencia CC BY-NC-ND (http://creativecommons.org/licenses/by-nc-nd/4.0/). 


\section{Introducción}

Clostridioides difficile es responsable de diarrea infecciosa y colitis pseudomembranosa, cuya incidencia y prevalencia se incrementó por el uso y abuso de antibióticos. ${ }^{1}$ La presentación clínica puede ser desde asintomática hasta generar megacolon tóxico; en 2011, la mortalidad por esta infección se incrementó a $6.4 \%$ en Estados Unidos. ${ }^{2}$ En la actualidad no solamente ocurre en hospitales, sino también en la comunidad. ${ }^{3}$ El diagnóstico se realiza en muestras fecales: en dos pasos con inmunoensayo para detectar toxinas A y $B$ y enzima glutamato deshidrogenasa (GDH) y la confirmación con la amplificación de ácidos nucleicos; o en un paso con la realización solo de esta última (prueba que resulta costosa y de difícil acceso).1,2,4

De acuerdo con la política de dos pasos, en un estudio realizado por Goldenberg et al. en 2010 se reportaron 500 muestras fecales; la detección de toxinas se consideró redundante cuando se observó $\mathrm{GDH}$, ya que esta se considera altamente sensible, si bien es poco específica. ${ }^{3,5}$ El objetivo del presente estudio fue corroborar si la detección de toxinas de Clostridioides difficile es redundante cuando se determina GDH.

\section{Métodos}

Se realizó un estudio observacional retrospectivo en un hospital privado de México, el cual cuenta con 95 camas censables. Se analizaron todas las muestras fecales con sospecha de infección por Clostridioides difficile de pacientes hospitalizados o ambulatorios de cualquier edad, de diciembre de 2016 a octubre de 2018. La determinación de toxinas A y B y GDH fue realizada mediante inmunocromatografía cualitativa con kit CerTest $^{\circledR}$ (Biotec SL Zaragoza, España), cuya sensibilidad y especificidad es mayor de $0.99 \%$, conforme las especificaciones del fabricante. Con la información recolectada, los datos fueron analizados en tasas y proporciones. Además, se determinó el rendimiento diagnóstico de la detección de GDH respecto a la de toxinas. Se realizó un análisis bayesiano para definir el cambio de la probabilidad preprueba acorde con el resultado de la detección de GDH. Las proporciones en la detección de GDH y toxinas $A$ y $B$ fueron comparadas mediante chi cuadrada con el programa estadístico en línea VassarStats.net. Se consideró significativamente estadístico un valor de $p<0.05$.
Tabla 1. Evaluación del rendimiento diagnóstico de la enzima glutamato deshidrogenasa (GDH)

\begin{tabular}{|l|c|c|c|c|c|c|}
\hline GDH & \multicolumn{2}{|c|}{$\begin{array}{c}\text { Toxinas A o B } \\
\text { positivas }\end{array}$} & \multicolumn{2}{|c|}{$\begin{array}{c}\text { Toxinas A y B } \\
\text { negativas }\end{array}$} & \multicolumn{2}{|l|}{ Total } \\
\hline Positiva & 54 & $(66.66 \%)$ & 27 & 33.33 & 81 & $(100 \%)$ \\
\hline Negativa & 6 & $(2.41 \%)$ & 242 & 97.58 & 248 & $(100 \%)$ \\
\hline Total & 60 & $(18.23 \%)$ & 269 & 81.76 & 329 & $(100 \%)$ \\
\hline
\end{tabular}

$\chi^{2}=164.7, p<0.001$. Prevalencia de $18.2 \%$ (IC $\left.95 \%=19.3-22.9\right)$,

sensibilidad de 0.90 , especificidad de 0.89 , valor predictivo positivo de 0.67 (IC $95 \%$

$=0.55-0.77$ ) valor predictivo negativo de 0.97 (IC $95 \%=0.95-0.99)$. cociente de probabilidad positivo de 8.9 (IC 95\% = 6.2-12.9, cociente de probabilidad negativo de 0.11 (IC $95 \%=0.05-0.24$ )

\section{Resultados}

Se evaluaron 329 resultados de análisis de toxinas A y B, así como de GDH; 176 correspondieron a mujeres $(53.4 \%)$ y solamente $47(14.2 \%)$ a pacientes hospitalizados. La mediana de la edad fue de 56 años (rango intercuartílico $=32-71$ ). Se detectó toxina $A$ en 52 especímenes (15.8\%), toxina B en $41(12.4 \%)$ y GDH en 81 (24.6\%).

En las muestras de 60 pacientes se detectó al menos una de las toxinas; por lo tanto, la prevalencia fue de $18.2 \%$. Se determinó que la GDH tenía una sensibilidad y especificidad de 0.90 y 0.89 , respectivamente. El valor predictivo positivo fue de $0.67 \mathrm{y}$ el valor predictivo negativo, de 0.97 . El cociente de probabilidad positivo fue de 8.9 , mientras que el cociente de probabilidad negativo, de 0.11 (Tabla 1). El análisis bayesiano determinó la probabilidad de presentar infección por Clostridioides difficile con la detección de GDH. Por lo anterior, si el resultado de GDH es positivo, la probabilidad pasa de 0.182 a 0.66 ; cuando el resultado es negativo, la probabilidad de infección pasa de 0.182 a 0.023 .

\section{Discusión}

En la institución donde se realizó el presente estudio se emplea el algoritmo de dos pasos, sin embargo, la implementación de la amplificación de ácidos nucleicos se efectúa esporádicamente debido a su elevado costo, por lo que el diagnóstico se lleva a cabo generalmente con el cuadro clínico y las pruebas de escrutinio.

La prevalencia de Clostridioides difficile determinada por la detección de cualquiera de las toxinas fue de $18.2 \%$ en la muestra estudiada. Esta proporción tal vez puede estar sub-representada debido a que la identificación de toxinas no es considerada el 
estándar de oro. La presencia de GDH aumenta la probabilidad posprueba de infección por Clostridioides difficile a $66 \%$ y cuando no es detectada, disminuye a 2.3\%. Lo anterior sugiere un adecuado rendimiento para descartar la infección cuando la detección de GDH resulta negativa.

Estos resultados confirman la observación de Goldenberg et al. respecto a la redundancia de la detectar toxinas junto con GDH. ${ }^{3,5}$ Consideramos que esta medida es necesaria en instituciones donde la amplificación de ácidos nucleicos no es accesible, debido a que se encontraron seis casos (10\%) con detección positiva de toxinas y negativa de GDH.

\section{Conflicto de intereses}

Los autores declaran no tener conflictos de intereses.

\section{Financiamiento}

El actual trabajo no recibió financiamiento de ningún tipo.

\section{Responsabilidades éticas}

Protección de personas y animales. Los autores declaran que para esta investigación no se realizaron experimentos en seres humanos ni en animales.

Confidencialidad de los datos. Los autores declaran que siguieron los protocolos de su centro de trabajo sobre la publicación de datos de pacientes.

Derecho a la privacidad y consentimiento informado. Los autores declaran que en este artículo no aparecen datos de pacientes.

\section{Bibliografía}

1. Bagdasarian N, Rao K, Malani PN. Diagnosis and treatment of clostridium difficile in adults: a systematic review. JAMA. 2015;313:398-408.

2. McDonald LC, Gerding DN, Johnson S, Bakken JS, Carroll KC, Coffin SE, et al. Clinical practice guidelines for clostridium difficile infection in adults and children: 2017 update by the Infectious Diseases Society of America (IDSA) and Society for Healthcare Epidemiology of America (SHEA). Clin Infect Dis. 2018;66:e1-e48.

3. Shin BM, Lee EJ, Moon JW, Lee SY. Evaluation of the VIDAS glutamate dehydrogenase assay for the detection of clostridium difficile. Anaerobe. 2016;40:68-72.

4. Planche T, Karunaharan N. Clostridium difficile. Medicine. 2017;45:634-638.

5. Goldenberg SD, Cliff PR, French GL. Glutamate dehydrogenase for laboratory diagnosis of Clostridium difficile infection. J Clin Microbiol. 2010;48(8):3050-3051 